\title{
Um Modelo de Otimização Multiobjetivo para Gerenciamento de Microgrids
}

\author{
José Eduardo Almeida de Alencar*, \\ Igor Rafael Santos da Silva*, \\ Vitor Augusto Correa Cortez Almeida*, \\ Ricardo de Andrade Lira Rabêlo* \\ * Departamento de Computação, Universidade Federal do Piauí, PI, \\ (e-mail: jeduardo824@gmail.com,yggor14rafa@hotmail.com, \\ vitoracortez@gmail.com, ricardoalr@ufpi.edu.br).
}

\begin{abstract}
In this paper, a preference-based multi-objective optimization model is presented, considering the real-time energy price to solve the problem of optimal scheduling of residential loads. It aims to minimize the cost associated with consumption and the inconvenience caused to consumers. The model was formalized using a nonlinear programming problem, subject to constraints related to energy consumption and operational aspects related to appliance categories. The computational resolution of the model used the NSGA-III genetical algorithm to determine the scheduling of the devices, renewable energy resources and the use of the storage system for the entire time horizon, considering consumer preferences. The results show that the proposed model and the technique used can minimize the total cost associated with energy consumption and the inconvenience of consumers without the need for manual intervention.

Resumo: Para minimizar o custo associado ao consumo e a inconveniência causada ao consumidor, este artigo apresenta um modelo de otimização multiobjetivo baseado em preferências, que considera o preço da energia em tempo real para resolver o problema do agendamento ótimo das cargas residenciais. O modelo foi formalizado como um problema de programação não linear, sujeito a restrições associadas ao consumo de energia e aspectos operacionais relacionados às categorias de eletrodomésticos. A resolução computacional do modelo utilizou o algoritmo genético NSGA-III para determinar o agendamento dos aparelhos, dos recursos de energia renovável e a utilização do sistema de armazenamento para todo o horizonte de tempo, considerando as preferências do consumidor. Os resultados mostram que a abordagem proposta minimiza o custo total associado ao consumo de energia e a inconveniência dos consumidores.
\end{abstract}

Keywords: Smart Grids, Demand Response, Microgrid, NSGA-III, Optimization

Palavras-chaves: Redes Inteligentes, Resposta da Demanda, Microgrid, NSGA-III, Otimização

\section{INTRODUÇÃO}

Com o crescimento da população global, a complexidade do fornecimento de energia aumenta. Em resposta, estudos e pesquisas sobre a eficiência e confiança operacional dos sistemas de energia elétrica são necessários para prevenir problemas como interrupções ao serviço de fornecimento de eletricidade ou o aumento de custos (Dai et al., 2017), (Li et al., 2015), (Althaher et al., 2015), dificuldades que crescem com o esgotamento das fontes de energia não renováveis ao redor do mundo (Siddaraj and Tangi, 2016).

Uma solução para auxiliar a superar tais problemas é incorporar infraestruturas de medição avançadas, combinadas com tecnologias de comunicação e informação e medidores inteligentes através de uma Rede Inteligente (SG, do inglês Smart Grid). Esses sistemas aplicam tecnologias de comunicação e informação (TCI) para melhorar a interação entre os dispositivos de um sistema elétrico de potência (SEP) e os consumidores conectados ao mesmo (Zhao et al., 2013). Tal interação pode ser usada pelos con- sumidores finais para melhorar seus padrões de consumo visando reduzir o seu custo associado.

Em Park et al. (2017) afirma-se que metodologias de controle de resposta da demanda e aparelhos inteligentes podem otimizar o uso de recursos elétricos de maneira eficiente. Seguindo esta linha, em Muratori et al. (2014), Parvania and Fotuhi-Firuzabad (2010) os autores, do ponto de vista de uma SG, definem a Resposta da Demanda (DR, do inglês Demand Response) como um programa que fornece incentivos e benefícios aos consumidores finais para que os mesmos mudem seus padrões de consumo de eletricidade em resposta a mudanças no preço da eletricidade ao longo do tempo ou quando a confiança no sistema está comprometida. Os programas de DR mais comuns são baseados em preço, no qual uma tarifa modelo é usada para ajudar o usuário a ajustar seus padrões de consumo em resposta ao desvio no preço da eletricidade.

Baseando-se na definição prévia de DR, este conceito pode ser bem adotado em microgrids. Uma microgrid (MG) pode ser descrita com um agregador de recursos de 
energia distribuídos (RED), recursos de energia renovável (RER), sistemas de armazenamento de energia (SAE) e cargas locais, que podem operar tanto conectadas à rede principal como em modo isolado (Martin-Martínez et al., 2016). A MG permite assim uma produção de energia mais confiável, eficiente e favorável ao ambiente graças ao aumento do desenvolvimento de geração distribuída (GD), especialmente por meio de RER assim como SAE distribuídos (Hatziargyriou, 2014), (Liang and Zhuang, 2014).

A gestão de energia numa MG tem sido estudada com várias abordagens em estudos recentes, como sobre a maximização de receita e minimização de poluição do ambiente (Mohan et al., 2015), (Mohammadi et al., 2014), melhoramento da performance dinâmica considerando aspectos econômicos (Mohan et al., 2016), otimização do custo de operação e a performance econômica (Wang et al., 2016), (Yunjia et al., 2017) e o aprimoramento na confiança da MG (Nikmehr and Ravadanegh, 2016), assim como problemas de DR, tais como Pop et al. (2018), Taniguchi et al. (2017), Zunnurain and Maruf (2017), Li et al. (2017), Jovanovic et al. (2016), Wu et al. (2015). Entretanto, um mecanismo de otimização dirigido por preferências e o agendamento de cargas residenciais considerando as diferentes características de operação de diferentes categorias de eletrodomésticos não foram bem analisados.

Como a programação dos eletrodomésticos, o agendamento dos RER e do SAE requer tempo e conhecimentos específicos do consumidor (Rastegar and Fotuhi-Firuzabad, 2015), e o agendamento da gestão residencial deve considerar as preferências do consumidor sobre o uso desses aparelhos além da variação do preço da eletricidade, neste artigo um modelo de programação multiobjetivo baseado em preferências é modelado para a gestão de energia numa MG. O modelo visa otimizar o custo do consumo e a satisfação do consumidor de maneira simultânea. Uma MG básica é analisada, na qual o lado da produção inclui um sistema de painéis fotovoltaicos (PF) como recurso de energia renovável e um SAE. O Algoritmo Genético de Classificação por Não Dominância III (NSGA-III, do inglês Non-Dominated Sorted Genetic Algorithm) (Jain and Deb, 2014) é aplicado para resolver o problema multiobjetivo proposto. Várias simulações, estudos de caso e estudos comparativos são elaborados para demonstrar a eficiência e viabilidade da metodologia proposta.

\section{METODOLOGIA}

Essa seção apresenta o modelo de otimização de DR multiobjetivo para administrar as cargas de todos os aparelhos, considerando o preço em tempo real (PTR) (Wang et al., 2013), as características operacionais dos aparelhos, os recursos de energia renovável e o sistema de armazenamento de energia.

\subsection{Modelagem do Problema}

A função $f 1$ usada no modelo multiobjetivo proposto, é formulada como:

$$
\begin{gathered}
\text { Minimizar } \sum_{i=1}^{N} E_{i} \sum_{t=1}^{T}\left(P r_{t} * C D A_{t, i}\right)^{2} \\
*\left(1-\left(C D R E R_{t, i}-C D S A E_{t, i}\right)^{2}\right)
\end{gathered}
$$

onde $N$ é o numero de eletrodomésticos; $E_{i}(i=1, \ldots, N)$ representa o vetor de consumo de energia dos aparelhos $i$ quando em operação; $T$ é o horizonte de tempo; $P r_{t}$ é o preço da eletricidade no tempo t. $C D A_{t, i}$ (Configuração Diária dos Aparelhos), $C D R E R_{t, i}$ (Configuração Diária dos Recursos de Energia Renovável) e $C D S A E_{t, i}$ (Configuração Diária do Sistema de Armazenamento de Energia) são matrizes binárias com as configuração diárias de operação dos eletrodomésticos, recursos de energia renovável e sistema de armazenamento de energia, respectivamente. $C D A_{t, i}$ se refere à matriz de agendamento das cargas com a seguinte configuração:

$$
C D A_{t, i}= \begin{cases}1, & \text { se o aparelho } i \text { está ligado no } \\ & \text { intervalo de tempo } t \\ 0, & \text { caso contrário. }\end{cases}
$$

$C D R E R_{t, i}$ se refere a matriz de planejamento do RER e tem a seguinte configuração:

$$
C D R E R_{t, i}= \begin{cases}1, & \text { se o aparelho } i \text { está consu- } \\ & \text { mindo energia dos RER no } \\ & \text { intervalo de tempo } t, \\ 0, & \text { caso contrário. }\end{cases}
$$

$C D R E R_{t, i}$ define o agendamento dos eletrodomésticos que consomem energia dos RER. Neste trabalho, os RER são compostos por um sistema de painéis fotovoltaicos (PF) instalados na casa do consumidor. A saída dos PF depende da temperatura das células e da irradiância solar na situação de ponto de energia máximo (PEM), expressada em (4) (Riffonneau et al., 2011). A temperatura da $m$ ésima célula do PF é calculada por (4), e a potência de saída do PF em cada intervalo de tempo $t$, com $t=$ $1, \ldots, T$, pode ser obtida por (5) (Riffonneau et al., 2011). A equação (6) corresponde a uma restrição de igualdade para garantir que o consumo de energia renovável dos eletrodomésticos seja menor ou igual à saída do sistema fotovoltaico.

$$
\begin{gathered}
T_{m}(t)=T_{a m p}+\frac{G_{T}(t)}{G_{T_{S T C}}} *(N O C T-20) \\
t=1, \ldots, T \\
P_{P F}(t)=\left(\left[P_{P F, S T C} * \frac{G_{T}(t)}{G_{T_{S T C}}} *\left(1-\gamma *\left(T_{m}(t)-T_{m S T C}\right)\right)\right]\right. \\
\left.* N_{P F s} * N_{P F p}\right), t=1, \ldots, T \\
\sum_{i=1}^{N} E_{i} * C D R E R_{t, i} \leq P_{P F}(t), t=1, \ldots, T
\end{gathered}
$$

$C D S A E_{t, i}$ se refere à matriz de agendamento do sistema de armazenamento de energia (SAE), definido como:

$$
C D S A E_{t, i}= \begin{cases}1, & \text { se o aparelho } i \text { está consu- } \\ & \text { mindo energia do SAE no in- } \\ & \text { tervalo de tempo } t \\ 0, & \text { caso contrário. }\end{cases}
$$


$C D S A E_{t, i}$ define o agendamento dos eletrodomésticos que utilizam energia do SAE. Neste trabalho, o SAE é composto por um sistema de baterias conectadas aos painéis fotovoltaicos $(\mathrm{PF})$ e instaladas na residência do consumidor. O SAE atua como um armazenador de energia elétrica gerada pelo sistema de PF, assim como fonte de energia para os aparelhos residenciais quando o preço da energia está elevado (Mathiesen et al., 2015). O modelo do sistema de armazenamento de energia é mostrado em (8)-(14)(Motevasel and Seifi, 2014).

$$
\begin{gathered}
P_{S A E}(t)=E_{A}(t)-E_{A}(t-1), t=1, \ldots, T \\
E_{A}^{m i n} \leq E_{A}(t) \leq E_{A}^{\max }, t=1, \ldots, T \\
E_{A}^{\text {min }}-E_{A}(0) \leq \sum_{k=1}^{t}\left(P_{S A E}(k)\right) \leq E_{A}^{\max }-E_{A}(0), \\
t=1, \ldots, T \\
E_{A}(0)=E_{A}(T) \\
-\omega_{C}^{E} * P_{S A E}(t) \leq P_{E-c h a r}^{\max }, t=1, \ldots, T \\
\frac{P_{S A E}(t)}{\omega_{D}^{E}} \leq P_{E-\text { disch }}^{\max }, t=1, \ldots, T \\
\sum_{i=1}^{N} E_{i} * C D S A E_{t, i} \leq P_{S A E}(t), t=1, \ldots, T
\end{gathered}
$$

onde $E_{A}(t)$ é a energia armazenada na bateria no instante $t ; P_{S A E}(t)$ é a potência de saída da bateria no instante $t$; $E_{A}^{m i n}$ e $E_{A}^{\max }$ são os limites máximo/mínimo de energia armazenada na bateria, respectivamente. (8) estabelece que a potência de saída da bateria não pode ser maior que a energia estocada no momento. (9) mostra que a energia nas baterias deve ser limitada entre os níveis de mínimo e máximo visando evitar a redução da vida útil das baterias. A cada intervalo de tempo $t$, o $P_{S A E}(t)$ deve estar entre esses limites. Carregamento e descarregamento de energia em cada instante $t$ são limitados pela energia armazenada na bateria, como mostrado em (10). O estado inicial e final da carga da bateria deve ser o mesmo descrito por (11). A limitação no carregamento/descarregamento para as baterias no SAE é exibido em (12) e (13). (14) define que o consumo de energia promovido pelo SAE deve ser menor ou igual a sua potência de saída.

A função $f 2$ mede a inconveniência e avalia como o agendamento otimizado dos eletrodomésticos pode modificar a satisfação/conforto do consumidor final e é dada por:

$$
\begin{gathered}
\text { Minimizar } \sum_{t=1}^{T} \sum_{i=1}^{N}\left(\operatorname{Incv}_{t, i}^{\text {horaria }}\left(C D A_{t, i}\right)\right. \\
\left.+\operatorname{Incv}_{t, i}^{\text {termica }}\left(C D A_{t, i}\right)\right)
\end{gathered}
$$

A avaliação do custo de consumo considera dois tipos de situações de inconveniência previamente definidas pelo consumidor. Quanto maior for o custo expresso por (15), maior será a inconveniência, indicando o grau de diferença entre o agendamento proposto e o desejado pelo consumidor. Essas situações são calculadas por meio dos horários de operação e condições térmicas de cada aparelho, conforme as configurações do consumidor.

A inconveniência é definida de duas maneiras: inconveniência horária e inconveniência térmica. A inconveniência horária calcula o custo da eletricidade associado ao consumo dos eletrodomésticos quando usados em horários inconvenientes, de acordo com o perfil operacional definido pelo consumidor. Tal perfil é composto por dois vetores, Perfil_Tempo e Perfil_Req, os quais permitem que os eletrodomésticos operem com múltiplos horários de início/término. Cada aparelho $i$ tem um vetor Tam_Perfil $l_{i}$, que representa o número de tempos de operação diferentes para aquele aparelho específico. Baseado nisso, a inconve-

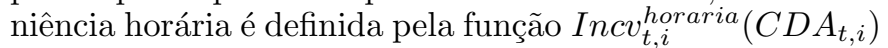
e é dada por:

$$
\begin{gathered}
\operatorname{Incv}_{t, i}^{\text {horaria }}\left(C D A_{t, i}\right)= \\
\begin{cases}P r_{t} *\left(T I_{i}^{j}-t\right) * C D A_{t, i}, & \text { se } t<T I_{i}^{j} \\
0, & \text { se } T I_{i}^{j} \leq t \leq T F_{i}^{j} \\
P r_{t} *\left(t-T F_{i}^{j}\right) * C D A_{t, i}, & \text { se } t>T F_{i}^{j}\end{cases}
\end{gathered}
$$

onde $j=1, \ldots$, Tam_Perfil $l_{i}$.

A inconveniência térmica calcula o custo da eletricidade associado ao consumo dos eletrodomésticos quando usados sob condições térmicas consideradas inconvenientes pelo consumidor. É dada pela função $\operatorname{Inc} v_{t, i}^{\text {termica }}\left(C D A_{t, i}\right) \mathrm{com}$ a seguinte configuração:

$$
\begin{aligned}
& \text { Incvertic } \text { termica }_{t}\left(C D A_{t, i}\right)=
\end{aligned}
$$

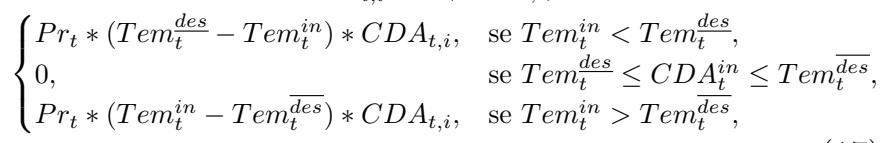

onde $T e m_{t}^{\frac{d e s}{t}}$ e $T e m_{t}^{\overline{d e s}}$ são as temperaturas internas mínima/máxima no tempo $t$, respectivamente; $\operatorname{Tem}_{t}^{i n}$ é a temperatura interna no tempo $t$, calculada como (Tsui and Chan, 2012):

$$
\begin{gathered}
\text { Tem }_{t}^{\text {interna }}=\text { Tem }_{t-1}^{\text {interna }}+\alpha *\left(\text { Tem }_{t}^{\text {externa }}-\text { Tem }_{t-1}^{\text {interna }}\right) \\
+\beta * C D A_{t}^{i} * E_{i}, t=1, \ldots, T, i=1, \ldots, N
\end{gathered}
$$

Onde Tem $_{t}^{\text {externa }}$ é a temperatura externa; $\alpha$ e $\beta$ são as condições térmicas envolvendo o eletrodoméstico térmico.

Com ambas funções objetivo definidas, a melhor solução é aquela na qual os aparelhos domésticos estão trabalhando o mais perto possível da situação desejada definida pelo consumidor e ao mesmo tempo reduzindo o custo associado ao consumo de eletricidade. Quanto mais perto o agendamento está do desejado, melhor a solução.

As funções objetivo $f 1$ e $f 2$ estão sujeitas a um conjunto de restrições. A Restrição 1 (19) estabelece os limites (mínimo e máximo) para os níveis de carga em cada intervalo de tempo $t$ : 


$$
d_{t}^{\min } \leq \sum_{i=1}^{N} C D A_{t, i} * P_{i} \leq d_{t}^{\max }, t=1, \ldots, T
$$

onde $d_{t}^{\text {min }}$ é a demanda mínima para os níveis de carga em cada intervalo de tempo $t ; P_{i}(i=1, \ldots, N)$ é o vetor com a potência (em $\mathrm{kW}$ ) de cada um dos eletrodomésticos; $d_{t}^{\max }$ é a demanda máxima para os níveis de carga em cada intervalo de tempo $t$.

A Restrição 2 (20) estabelece que a mudança de carga entre horas adjacentes não deve exceder os limites de rampa inferiores/superiores:

$r^{D} \leq \sum_{i=1}^{N}\left(C D A_{t+1, i}-C D A_{t+1, i}\right) * P_{i} \leq r^{U}, t=1, \ldots, T-1$,

onde $r^{D}$ e $r^{U}$ correspondem aos limite de rampa máximo e mínimo para o intervalo de tempo $t$, respectivamente.

Restrição 3 (21) define o consumo de eletricidade diário mínimo $($ cedm):

$$
\sum_{i=1}^{N} \sum_{t=1}^{T} C D A_{t, i} * E_{i} \geq c e d m
$$

As restrições 1-3 (19-21) referem-se às características comuns de consumo de energia. Como foi previamente mencionado, a operação dos eletrodomésticos com múltiplos horários de início/término deve ser permitida, logo é necessário definir algumas restrições quanto à operação desses aparelhos.

Para controlar a operação dos eletrodomésticos, dois vetores chamados Perfil_Tempo e Perfil_Req, foram definidos. Perfil_Tempo $(22)$ indica um vetor de pares no formato (horário de início, horário de término), para cada aparelho residencial $i, i=1, \ldots, N$ :

$$
\text { Perfil_Tempo }{ }_{i}=\left[\left(T I_{1}, T F_{1}\right), \ldots,\left(T I_{j}, T F_{j}\right)\right]
$$

onde $j=1, \ldots$, Tam_Perfil $l_{i}$.

Já Perfil_Req $q_{i}$ (23) representa um vetor numérico, cujos valores representam o tempo de operação de cada eletrodoméstico $i$, com $i=1, \ldots, N$, de acordo com os horários de início e término definidos em Perfil_Tempo dos respectivos eletrodomésticos:

$$
\text { Perfil_Req } q_{i}=\left(\operatorname{Req}_{1}, \ldots, \operatorname{Req}_{j}\right)
$$

onde $j=1, \ldots$, Tam_Perfil $i l_{i}$.

As seguintes restrições conectam e limitam os vetores Perfil_Tempo e Perfil_Req. (24) define o tamanho dos vetores. (25) e (26) respectivamente, relacionam os valores de ambos vetores e evitam sobreposição dos horários de operação; (27) e (28) definem que o tempo de operação máximo de cada eletrodoméstico não deve ser maior que o horizonte de tempo.

$$
\begin{gathered}
\mid \text { Perfil_Tempo }|=| \text { Perfil_Req } \mid, \\
\mid \text { Perfil_Req } \mid=\text { Tam_Perfil } l_{i}, \\
i=1, \ldots, N \\
T F_{i}^{j}-T I_{i}^{j} \geq \text { Req } q_{i}^{j}, \\
i=1, \ldots, N, j=1, \ldots, \text { Tam_Perfil } i
\end{gathered}
$$

$$
\begin{gathered}
T F_{i}^{j} \leq T I_{i}^{j+1}, \\
i=1, \ldots, N, j=1, \ldots, \text { Tam_Perf } i l_{i}-1 \\
\sum_{j=1}^{T a m_{-} \text {Perfil }}{ }^{2} \operatorname{Req}_{i}^{j} \leq T, i=1, \ldots, N \\
T F_{i}^{\text {Tam_Perfil }}{ }_{i}-T I_{i}^{1} \leq T, i=1, \ldots, N
\end{gathered}
$$

Neste trabalho, os eletrodomésticos são divididos em três categorias baseadas nas suas características operacionais Chen et al. (2012) que são: interruptível e adiável $\left(A_{I}\right)$; ininterruptível e adiável $\left(A_{I I}\right)$; e ininterruptível e nãoadiável $\left(A_{I I I}\right)$. Ininterruptível se refere a uma operação que não pode ser interrompida até ser completada. Adiável e não-adiável definem que a operação deve começar no primeiro intervalo de tempo definido como horário de início do eletrodoméstico, ou não começar. As restrições que lidam com as diferentes categorias de eletrodomésticos $\left(A_{I}\right)$, $\left(A_{I I}\right)$ e $\left(A_{I I I}\right)$ são baseada nas definições especificadas acima.

Restrição 5 (29) estabelece que a inicialização operacional de cada eletrodoméstico da categoria $A_{I}$ pode variar sobre o tempo definido sobre cada par $\left(T I_{i}^{j}, T F_{i}^{j}\right)$ definido pelo consumidor, fazendo com que $R e q_{i}^{j}$ seja respeitado:

$$
\sum_{t=T I_{i}^{j}}^{T F_{i}^{j}} C D A_{t, i} \geq R e q_{i}^{j}, i \in A_{I}, j=1, \ldots, \text { Tam_Perfi } \chi_{i}(29)
$$

onde $R e q_{i}^{j}$ o tempo necessário para o eletrodoméstico $i$ terminar sua operação no intervalo definido por $\left(T I_{i}^{j}, T F_{i}^{j}\right)$; $A_{I}$ é um conjunto de índices dos aparelhos categorizados como interruptíveis e adiáveis.

Restrição 6 (30) define que a inicialização operacional dos eletrodomésticos da categoria $A_{I I}$ pode ser atrasado no intervalo $\left(T I_{i}^{j}, T F_{i}^{j}\right)$, mas, depois de começado, não pode ser interrompido:

$$
\begin{gathered}
\sum_{q=T I_{i}^{j}}^{T F_{i}^{j}-R e q_{i}^{j}} \prod_{t=q}^{R e q_{i}+q} C D A_{t, i} \geq 1, \\
\forall_{i} \in A_{I I}, j=1, \ldots, \text { Tam_Perfil } i l_{i}
\end{gathered}
$$

onde $q$ é o intervalo de tempo inicial do intervalo que será verificado se os eletrodomésticos da categoria $A_{I I}$ foram usados. $A_{I I}$ é o conjunto dos índices dos eletrodomésticos da categoria ininterruptíveis e adiáveis.

Restrição 7 (31) estabelece que a operação dos eletrodomésticos da categoria $A_{I I I}$ deve ser entre seu começo $\left(T I_{i}\right)$ e fim $\left(T F_{i}\right)$, como definindo pelo consumidor. É ininterruptível e não adiável para o intervalo de tempo $R e q_{i}$ no horizonte de tempo $T$ :

$$
\prod_{q=T I_{i}^{j}}^{T F_{i}^{j}} C D A_{t, i} \geq 1, \forall_{i} \in A_{I I I}, j=1, \ldots, \text { Tam_Perfil }(31)
$$


onde $T I_{i}$ é o tempo inicial da operação; $T F_{i}$ é o tempo final da operação; $A_{I I I}$ é o conjuntos dos índices do aparelhos categorizados como ininterruptíveis e não-adiáveis.

\section{RESULTADOS E DISCUSSÃO}

Nesta seção, os resultados das simulações computacionais são apresentadas visando avaliar o desempenho do modelo de otimização multiobjetivo proposto para DR usando a técnica de otimização NSGA-III restrito. Esse algoritmo foi escolhido por se adequar bem à resolução de problemas combinatoriais com muitos objetivos e restrições, mas com a vantagem de manter a diversidade das soluções em relação ao seu predecessor, o NSGA-II (Jain and Deb, 2014).

\subsection{Estudo de Caso}

No cenário de simulação, famílias compostas por 02 adultos sem crianças foram consideradas. O padrão de consumo de energia elétrica de cada família foi obtido através da ferramenta Load Profile Generator (LPG) (Pflugradt, 2016) para 03 famílias brasileiras vivendo nas cidades de Cuiabá-MT, São Paulo-SP e Teresina-PI localizadas respectivamente nas regiões Centro Oeste, Sudeste e Nordeste do Brasil.

Um horizonte de tempo $T$ com discretização por hora foi usado nas simulações computacionais que incluem os dias com maiores e menores consumo de energia elétrica de cada família entre 1 de Janeiro de 2016 e 31 de Dezembro de 2016. Assume-se que o todo intervalo de tempo do agendamento consiste de 24 subintervalos, ou seja, $t=\{1,2, \ldots, 24\}$. O preço do consumo unitário de energia em cada intervalo de tempo, ilustrado na figura 1, é baseado nos valores do Mercado de Eletricidade de Portugal (OMIE) para calcular o preço da eletricidade a cada hora, já que o Brasil não utiliza um programa de DR baseado em preços em tempo-real (PTR). O Sistema de Controle de Energia Residencial (SCER) proposto em (Veras et al., 2018) foi usado como uma arquitetura, na qual o modelo de DR proposto nesse artigo é responsável por determinar o agendamento das cargas e o ciclo de carga/descarga do SAE.

\subsection{Resultado das Simulações}

Neste trabalho, o esquema de ponto referência foi usado para achar apenas algumas soluções numa parte preferida da fronteira ótima de Pareto (Jain and Deb, 2014). Esse esquema atua como uma maneira de representar as preferências do consumidor no processo de otimização, ajudando na tomada de decisão.

Como definido por Jain e Deb em (Jain and Deb, 2014), para achar um conjunto preferido de soluções, um conjunto de pontos de referência $(R p)$ na região de preferência do consumidor deve ser dado. Em adição, $M$ pontos de referência extremos $(1,0)^{T},(0,1)^{T}$ são incluídos para fazer com que o preço de normalização trabalhe e melhor e faça um total de $|R p|+M$ pontos de referência, isto é o conjunto $P$ de pontos de referência. Esses pontos extremos são necessários para garantir que os pontos nadir e ideais Lu and Anderson-Cook (2013) Zitzler et al. (2007)

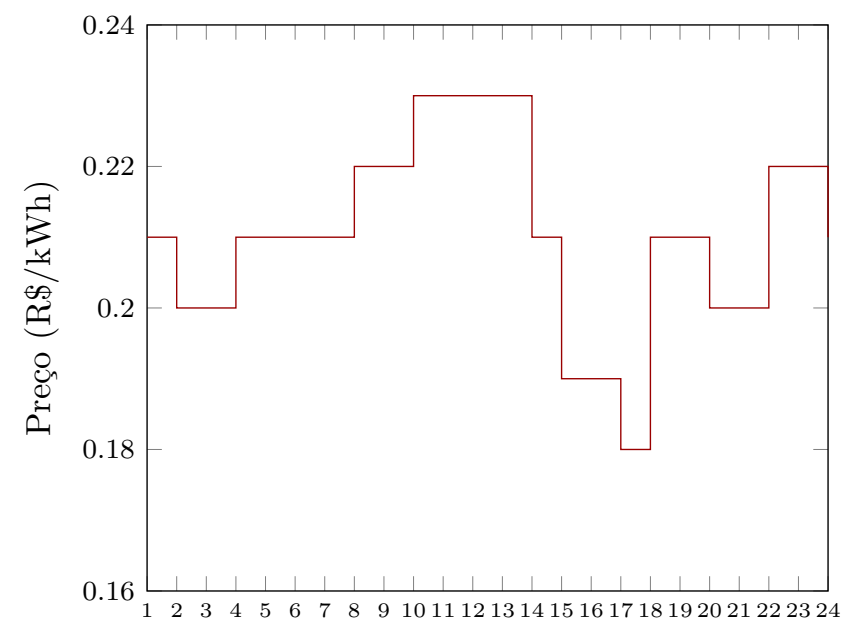

Subintervalos de tempo

Figura 1. Preço por unidade de potência consumida

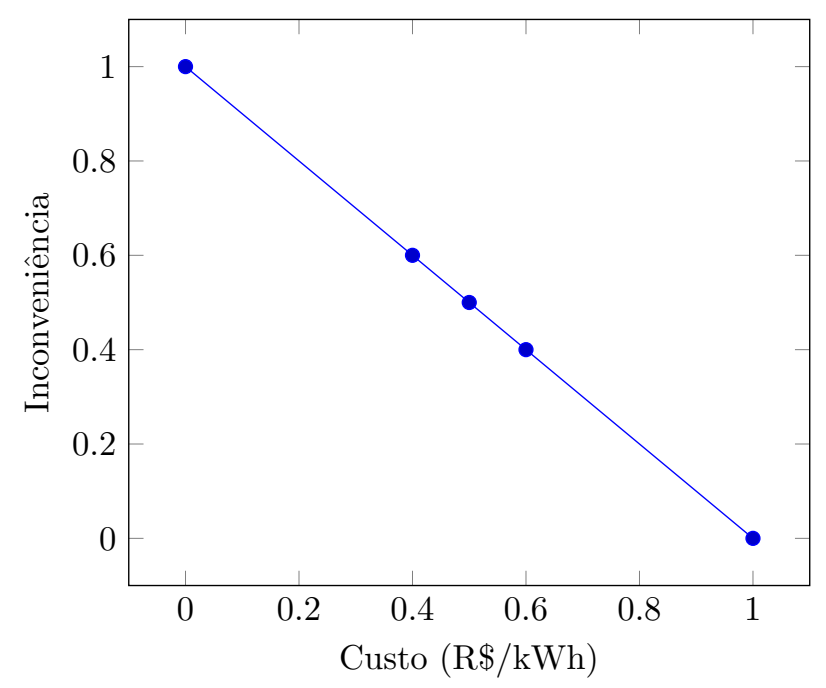

Figura 2. Um conjunto de pontos de referência preferidos encontra as soluções ótimas de Pareto correspondentes para o problema de DR

dos membros da população são adequadamente calculados para o propósito da normalização no algoritmo NSGA-III. Nessas simulações, três pontos de referência (conjunto $R p$ ) são usados, no meio do hiper-plano normalizado, como mostrado na figura 2.

Conforme mencionado anteriormente, dois pontos extremos a mais (conjunto $M$ ) são adicionados para fazer um total de cinco pontos de referência (conjunto $H$ ). Para o crossover e probabilidade de mutação foram usados os valores 0,6 e 0,1 respectivamente. A população tem 8 cromossomos e o número máximo de iterações foi 700 . Na figura 3, as soluções ótimas de Pareto não normalizadas obtidas são mostradas. Essas soluções foram analisadas considerando o padrão de consumo de energia de cada família, obtido através do LPG.

A tabela 1 apresenta os resultados das simulações obtidos para cada família, levando em conta o ponto extremo relacionado com o objetivo de minimização do custo, que é relacionado com a solução ótima de Pareto para esse obje- 


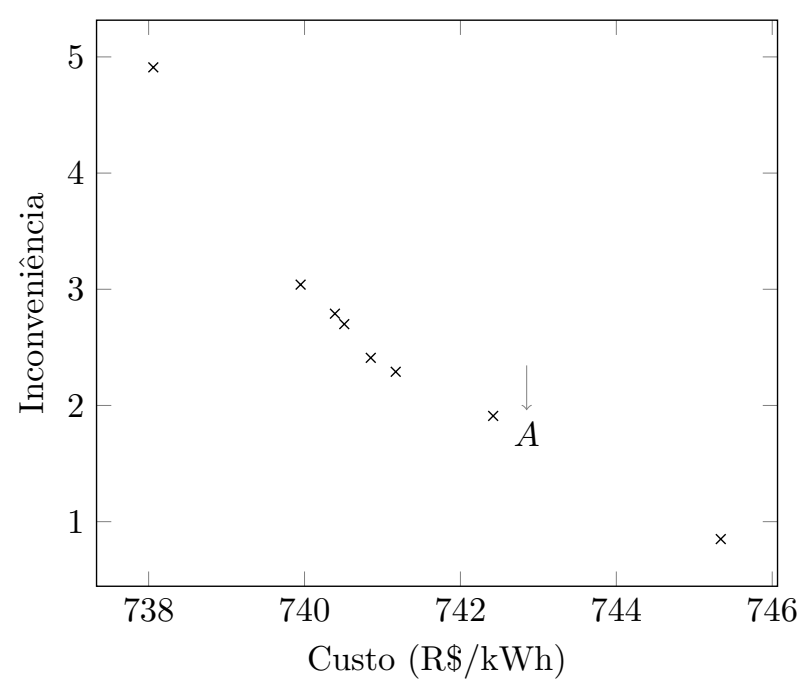

Figura 3. Soluções ótimas de Pareto não normalizadas

tivo. Portanto, é possível observar que a família residente na cidade de São Paulo obteve o melhor resultado em relação à redução total do custo de consumo de eletricidade, partindo de $\mathrm{R} \$ 301,64$ para $\mathrm{R} \$ 220,36$, totalizando uma redução de $26,95 \%$.

Tabela 1. Redução de custo ótima por cidade

\begin{tabular}{cccc}
\hline Cidade & $\begin{array}{c}\text { Custo Sem } \\
\text { DR }(\mathbf{R} \$)\end{array}$ & $\begin{array}{c}\text { Custo Com } \\
\text { DR }(\mathbf{R} \$ \mathbf{)}\end{array}$ & $\begin{array}{c}\text { Redução } \\
\mathbf{( \% )}\end{array}$ \\
\hline Cuiabá & 345,21 & 258,23 & 25,20 \\
Teresina & 346,39 & 259,09 & 25,20 \\
São Paulo & 301,64 & 220,36 & 26,95 \\
\hline
\end{tabular}

A tabela 2 apresenta os resultados das simulações obtidos para cada família levando em conta o ponto extremos relacionado com o objetivo de minimização da inconveniência, que é relacionado com a solução ótima de Pareto para esse objetivo. Logo, é possível observar que família residente na cidade de São Paulo obteve a menor inconveniência associada ao custo, com o valor de $\mathrm{R} \$ 0,27$.

Tabela 2. Inconveniência ótima por cidade

\begin{tabular}{cc}
\hline Cidade & Inconveniência $\mathbf{( R} \$ \mathbf{)}$ \\
\hline Cuiabá & 0,30 \\
Teresina & 0,28 \\
São Paulo & 0,27 \\
\hline
\end{tabular}

A solução $A$ destacada na figura 3 foi a solução mais próxima do ponto ótimo $(0,0)$. Essa solução apresenta a melhor relação entre os valores dos dois objetivos formulados no problema de DR apresentado nesse artigo. A cidade de São Paulo obteve o melhor resultado para o objetivo $f 1$, minimizar o custo, com um decaimento total de $26,7 \%$ no custo de consumo. Já para o objetivo $f 2$, minimizar a inconveniência, a cidade de Teresina obteve o menor custo associado à inconveniência, no valor de $\mathrm{R} \$$ 0,80 . Os resultados obtidos na solução $A$ são apresentados nas tabelas 3 e 4 .

\subsection{Impacto dos RER e SAE}

O impacto do RER e do SAE nos resultados obtidos também foram analisados. Um cenário sem o sistema
Tabela 3. Redução de custo da solução $A$ por cidade

\begin{tabular}{cccc}
\hline Cidade & $\begin{array}{c}\text { Custo Sem } \\
\text { DR } \mathbf{( R \$ )}\end{array}$ & $\begin{array}{c}\text { Custo Com } \\
\text { DR }(\mathbf{R} \$ \mathbf{)}\end{array}$ & $\begin{array}{c}\text { Redução } \\
\mathbf{( \% )}\end{array}$ \\
\hline Cuiabá & 345,21 & 259,35 & 24,8 \\
Teresina & 346,39 & 260,06 & 24,9 \\
São Paulo & 301,64 & 221,09 & 26,7 \\
\hline
\end{tabular}

Tabela 4. Inconveniência da solução $A$ por cidade

\begin{tabular}{cc}
\hline Cidade & Inconveniência (R\$) \\
\hline Cuiabá & 0,82 \\
Teresina & 0,80 \\
São Paulo & 0,81 \\
\hline
\end{tabular}

fotovoltaico e SAE foi simulado e o modelo de DR foi adaptado para lidar com essas mudanças. A equação 1 foi mudada para o seguinte:

$$
\text { Minimizar } \sum_{i=1}^{N} E_{i} \sum_{t=1}^{T}\left(P r_{t} * C D A_{t, i}\right)^{2}
$$

As restrições (4-6) e (8-14) não foram consideradas nessa simulação. A solução ótima de Pareto relacionada com o ponto extremo do objetivo de minimização do custo de ambas simulações foi comparada. A tabela 5 mostra que o custo ótimo da nova simulação é, ao menos, 13,4\% maior que o custo ótimo obtido nas simulações prévias, como ocorrido na cidade de Cuiabá, com um incremento de $\mathrm{R} \$$ 34,79 no custo de consumo, enquanto a cidade de Teresina obteve um aumento de 15,2\% no custo de eletricidade associado ao consumo, totalizando $\mathrm{R} \$ 39,60$.

Tabela 5. Comparação entre cenário com e sem RER e SAE

\begin{tabular}{cccc}
\hline Cidade & $\begin{array}{c}\text { Com RER e } \\
\text { SAE }(\mathbf{R} \$)\end{array}$ & $\begin{array}{c}\text { Sem RER e } \\
\text { SAE }(\mathbf{R} \$)\end{array}$ & $\begin{array}{c}\text { Aumento } \\
(\%)\end{array}$ \\
\hline Cuiabá & 258,23 & 293,02 & 13,4 \\
Teresina & 259,99 & 299,10 & 15,4 \\
São Paulo & 220,36 & 251,49 & 14,1 \\
\hline
\end{tabular}

\section{CONCLUSÃO}

Este artigo propõe um modelo de otimização de DR multiobjetivo para gerenciar a programação de eletrodomésticos de diversas categorias em um ambiente de microgrids, visando minimizar o custo associado ao consumo de eletricidade, bem como minimizar os inconvenientes (insatisfação/desconforto) dos consumidores finais, considerando recursos energéticos renováveis (RRES) e um sistema de armazenamento de energia (ESS). A programação de eletrodomésticos em redes inteligentes permite que o EPS seja mais eficiente e eficaz, pois problemas como interrupções de energia durante picos de demanda podem ser minimizados. Assim, a DR desempenha um papel fundamental na gestão do consumo de energia, a fim de evitar despesas gerais e reduzir os custos associados ao consumo de eletricidade para os consumidores finais.

O desempenho do modelo de otimização de DR proposto foi avaliado por meio de simulações. Primeiramente, foi analisada a eficiência para minimização dos custos associados ao consumo de energia elétrica, bem como a minimização das inconveniências (insatisfação/desconforto) aos 
consumidores finais, considerando as preferências informadas pelo consumidor. Além disso, o modelo multiobjetivo foi adaptado para lidar com o mesmo problema de DR sem considerar os sistemas RRES e ESS, a fim de verificar a influência desses recursos na redução do custo associado ao consumo de eletricidade. Os resultados do estudo mostraram que há uma redução significativa no custo total associado ao consumo de energia elétrica, aliada a um baixo custo associado ao inconveniente causado a todas as famílias consideradas no cenário de simulação.

Pesquisas futuras poderiam incluir veículos elétricos e mais recursos renováveis para a geração de energia elétrica no modelo de otimização. Outra direção poderia ser minimizar a poluição ambiental como um objetivo no modelo de otimização. Uma terceira opção é implementar o modelo proposto em um monitor em casa, de modo a usar a proposta em um cenário de computação de borda.

\section{REFERÊNCIAS}

Althaher, S., Mancarella, P., and Mutale, J. (2015). Automated demand response from home energy management system under dynamic pricing and power and comfort constraints. IEEE Transactions on Smart Grid, 6(4), 1874-1883.

Chen, Z., Wu, L., and Fu, Y. (2012). Real-time price-based demand response management for residential appliances via stochastic optimization and robust optimization. IEEE Transactions on Smart Grid, 3(4), 1822-1831.

Dai, Y., Gao, Y., Gao, H., and Zhu, H. (2017). Realtime pricing scheme based on stackelberg game in smart grid with multiple power retailers. Neurocomputing, 260, $149-156$.

Hatziargyriou, N. (2014). Microgrids: architectures and control. John Wiley \& Sons.

Jain, H. and Deb, K. (2014). An evolutionary manyobjective optimization algorithm using reference-point based nondominated sorting approach, part ii: Handling constraints and extending to an adaptive approach. IEEE Transactions on Evolutionary Computation, 18(4), 602-622.

Jovanovic, R., Bousselham, A., and Bayram, I.S. (2016). Residential demand response scheduling with consideration of consumer preferences. Applied Sciences, 6(1), $16-29$.

Li, C., Yu, X., Yu, W., Chen, G., and Wang, J. (2017). Efficient computation for sparse load shifting in demand side management. IEEE Transactions on Smart Grid, 8(1), 250-261.

Li, W.T., Yuen, C., Hassan, N.U., Tushar, W., Wen, C.K., Wood, K.L., Hu, K., and Liu, X. (2015). Demand response management for residential smart grid: From theory to practice. IEEE Access, 3, 2431-2440.

Liang, H. and Zhuang, W. (2014). Stochastic modeling and optimization in a microgrid: A survey. Energies, 7(4), 2027-2050.

Lu, L. and Anderson-Cook, C.M. (2013). Adapting the hypervolume quality indicator to quantify trade-offs and search efficiency for multiple criteria decision making using pareto fronts. Quality and Reliability Engineering International, 29(8), 1117-1133.

Martin-Martínez, F., Sánchez-Miralles, A., and Rivier, M. (2016). A literature review of microgrids: A functional layer based classification. Renewable and Sustainable Energy Reviews, 62, 1133-1153.

Mathiesen, B.V., Lund, H., Connolly, D., Wenzel, H., Østergaard, P.A., Möller, B., Nielsen, S., Ridjan, I., Karnøe, P., Sperling, K., et al. (2015). Smart energy systems for coherent $100 \%$ renewable energy and transport solutions. Applied Energy, 145, 139-154.

Mohammadi, S., Soleymani, S., and Mozafari, B. (2014). Scenario-based stochastic operation management of microgrid including wind, photovoltaic, micro-turbine, fuel cell and energy storage devices. International Journal of Electrical Power \& Energy Systems, 54, 525-535.

Mohan, V., Singh, J.G., and Ongsakul, W. (2015). An efficient two stage stochastic optimal energy and reserve management in a microgrid. Applied energy, 160, 28-38.

Mohan, V., Singh, J.G., Ongsakul, W., MP, R.S., et al. (2016). Economic and network feasible online power management for renewable energy integrated smart microgrid. Sustainable Energy, Grids and Networks, 7, 1324.

Motevasel, M. and Seifi, A.R. (2014). Expert energy management of a micro-grid considering wind energy uncertainty. Energy Conversion and Management, 83, $58-72$.

Muratori, M., Schuelke-Leech, B.A., and Rizzoni, G. (2014). Role of residential demand response in modern electricity markets. Renewable and Sustainable Energy Reviews, 33, 546-553.

Nikmehr, N. and Ravadanegh, S.N. (2016). Reliability evaluation of multi-microgrids considering optimal operation of small scale energy zones under load-generation uncertainties. International Journal of Electrical Power E Energy Systems, 78, 80-87.

Park, L., Jang, Y., Cho, S., and Kim, J. (2017). Residential demand response for renewable energy resources in smart grid systems. IEEE Transactions on Industrial Informatics, 13(6), 3165-3173.

Parvania, M. and Fotuhi-Firuzabad, M. (2010). Demand response scheduling by stochastic scuc. IEEE Transactions on smart grid, 1(1), 89-98.

Pflugradt, N.D. (2016). Modellierung von Wasser und Energieverbräuchen in Haushalten. Master's thesis, Technische Universitat Chemnitz, Chemnitz.

Pop, C., Cioara, T., Antal, M., Anghel, I., Salomie, I., and Bertoncini, M. (2018). Blockchain based decentralized management of demand response programs in smart energy grids. Sensors, 18(1), 162-182.

Rastegar, M. and Fotuhi-Firuzabad, M. (2015). Outage management in residential demand response programs. IEEE Transactions on Smart Grid, 6(3), 1453-1462.

Riffonneau, Y., Bacha, S., Barruel, F., Ploix, S., et al. (2011). Optimal power flow management for grid connected pv systems with batteries. IEEE Transactions on Sustainable Energy, 2(3), 309-320.

Siddaraj, U. and Tangi, S. (2016). Integration of dg systems composed of photovoltaic and a micro-turbine in remote areas. In Computation of Power, Energy Information and Commuincation (ICCPEIC), 2016 International Conference on, 005-008. IEEE.

Taniguchi, Y., Kutsuzawa, R., Yamashita, A., Matsumoto, J., and Yamanaka, N. (2017). Demand response minimizing the impact on the consumers' utility considering forecast error on renewable energy resource. In IEEE In- 
ternational Symposium on Local and Metropolitan Area Networks (LANMAN), 2017, 1-6. IEEE.

Tsui, K.M. and Chan, S.C. (2012). Demand response optimization for smart home scheduling under real-time pricing. IEEE Transactions on Smart Grid, 3(4), 18121821.

Veras, J.M., Silva, I.R.S., Pinheiro, P.R., Rabêlo, R.A.L., Veloso, A.F.S., Borges, F.A.S., and Rodrigues, J.J.P.C. (2018). A multi-objective demand response optimization model for scheduling loads in a home energy management system. Sensors, 18(10). doi:10.3390/s18103207. URL http: //www . mdpi . com/1424-8220/18/10/3207.

Wang, C., Liu, Y., Li, X., Guo, L., Qiao, L., and Lu, H. (2016). Energy management system for standalone diesel-wind-biomass microgrid with energy storage system. Energy, 97, 90-104.

Wang, Z., Paranjape, R., Sadanand, A., and Chen, Z. (2013). Residential demand response: An overview of recent simulation and modeling applications. In 26th Annual Canadian Conference on Electrical and Computer Engineering (CCECE'13, 1-6. Regina, Canada.

Wu, Z., Tazvinga, H., and Xia, X. (2015). Demand side management of photovoltaic-battery hybrid system. Applied Energy, 148, 294-304.

Yunjia, L., Tenglong, M., Jialiang, L., and Zhanzhan, Q. (2017). Independent microgrid day-ahead optimization based on demand response. In 29th Chinese Control And Decision Conference (CCDC), 2017, 5809-5814. IEEE.

Zhao, Z., Lee, W.C., Shin, Y., and Song, K.B. (2013). An optimal power scheduling method for demand response in home energy management system. IEEE Transactions on Smart Grid, 4(3), 1391-1400.

Zitzler, E., Brockhoff, D., and Thiele, L. (2007). The hypervolume indicator revisited: On the design of paretocompliant indicators via weighted integration. In Evolutionary multi-criterion optimization, 862-876. Springer.

Zunnurain, I. and Maruf, M.N.I. (2017). Automated demand response strategies using home energy management system in a res-based smart grid. In 4th International Conference on Advances in Electrical Engineering (ICAEE), 2017, 664-668. IEEE. 